\title{
Troponin utilization in patients presenting with atrial fibrillation/flutter to the emergency department: retrospective chart review
}

\author{
Nazanin Meshkat ${ }^{*}$, Emily Austin ${ }^{2}$, Rahim Moineddin ${ }^{3}$, Hamidreza Hatamabadi $^{4}$, Behzad Hassani ${ }^{5}$, Ali Abdalvand ${ }^{6}$ \\ and Amanda Marcuzzi ${ }^{7}$
}

\begin{abstract}
Background: There are few recommendations about the use of cardiac markers in the investigation and management of atrial fibrillation/flutter. Currently, it is unknown how many patients with atrial fibrillation/flutter undergo troponin testing, and how positive troponin results are managed in the emergency department. We sought to look at the emergency department troponin utilization patterns.

Methods: We performed a retrospective chart review of patients with atrial fibrillation/flutter presenting to the emergency department at three centers. Outcome measures included the rates of troponins ordered by emergency doctors, number of positive troponins, and those with positive troponins treated as acute coronary syndrome (ACS) by consulting services.
\end{abstract}

Results: Four hundred fifty-one charts were reviewed. A total of 388 (86\%) of the patients had troponins ordered, 13.7\% had positive results, and $4.9 \%$ were treated for ACS.

Conclusions: Troponin tests are ordered in a high percentage of patients with atrial fibrillation/flutter presenting to emergency departments. Five percent of our total patient cohort was diagnosed as having acute coronary syndrome by consulting services.

\section{Introduction}

The prevalence of atrial fibrillation is estimated at $0.1 \%$ among adults younger than 55 years, rising with age to $9 \%$ in those older than 80 [1,2], with clear implications in resource utilization by an aging population. It is estimated that the number of patients with atrial fibrillation will rise to about 5.6 million in 2050 from its current number of 2.3 million cases [3]. Atrial fibrillation is a resource-intensive arrhythmia as $70-80 \%$ of these patients are admitted during the course of their illness [4]. More importantly, a number of studies have shown that the hospitalization rates for atrial fibrillation have increased, up to 2-3 fold in the last 2 decades $[5,6]$.

Though there are few recommendations about the use of cardiac markers in the investigation and management

\footnotetext{
* Correspondence: nmeshkat@rogers.com

'Department of Medicine, Division of Emergency Medicine, University of

Toronto, Toronto, Canada

Full list of author information is available at the end of the article
}

of atrial fibrillation $[7,8]$, these tests are often ordered to diagnose myocardial infarction as the cause or consequence of the arrhythmia in patients with atrial fibrillation or flutter that present to emergency departments. Unnecessary use of cardiac markers may contribute to emergency department laboratory costs, delays in patient discharge if using serial tests, and unnecessary admission and cardiac investigations for positive troponins of unknown significance. Currently, it is unknown how many patients with atrial fibrillation/flutter undergo troponin testing and how positive troponin results are managed in the emergency department.

We sought to look at the rates of troponins ordered by emergency physicians, the rates of positive troponins, and how many of those with positive troponins were diagnosed as acute coronary syndrome by admitting services. To our knowledge, no studies have looked at the use of troponins in atrial fibrillation/flutter or the impact of positive troponins on the management of these patients.

\section{SpringerOpen ${ }^{\circ}$}

(c) 2011 Meshkat et al; licensee Springer. This is an Open Access article distributed under the terms of the Creative Commons Attribution License (http://creativecommons.org/licenses/by/2.0), which permits unrestricted use, distribution, and reproduction in any medium, provided the original work is properly cited. 


\section{Methods}

\section{Patient population}

A retrospective chart review was conducted at three Canadian centers: two academic emergency departments (AED 1 and 2) and one community emergency department (CED). A list of consecutive patients with new onset, paroxysmal or permanent atrial fibrillation, or atrial flutter that presented to the emergency departments during a 12-month period between January and December 2008 were generated by the health records departments at each center. All patients with the primary and secondary diagnosis of atrial fibrillation and atrial flutter were included. Exclusion criteria were as follows: patients presenting for INR checks and those directly admitted to a consulting service.

Research ethics board approval was obtained at all three sites.

\section{Sample size}

To aim for a 95\% confidence interval with a width less than $10 \%$ in the estimation of the rates of troponin testing and those treated for acute coronary syndrome, we reviewed a total of 450 charts (150 charts at each site). The charts reviewed were selected by systematic sampling from the list generated by the health record departments (described above). Charts with missing data were deleted.

\section{Study protocol}

Three blinded data abstractors (BH, EA, AM) were trained by the principal investigator to abstract data using a reference manual. Data were recorded in a formatted electronic database. A pilot chart review consisting of 15 charts per center was conducted prior to the study to streamline data extraction. The principal investigator performed periodic random checks, and meetings were scheduled to resolve any disputed data. The intra-rater reliability was determined using the Cohen's kappa rating, aiming for a minimum inter-rater reliability of $80 \%$.

All three sites used Troponin I (cTnI) as their cardiac marker. Two of the sites used the Beckman Coulter TnI immunoassay, and one site used the Abbott Architect TnI immunoassay. The cutoff troponin levels used were the respective coefficient variations of $10 \%$ for the specific immunoassays.

\section{Outcome measures}

We looked at the rate of troponin tests ordered by emergency doctors, those with positive troponins, and those diagnosed with acute coronary syndrome by the consulting services (defined as being given the diagnosis of acute coronary syndrome in the chart by the staff cardiologist or internist). Patients whose charts indicated positive troponins were reviewed to determine if alternate reasons for elevated troponin levels other than acute coronary syndrome had been documented. We also looked at discharge and referral patterns, emergency department (ED) visit length of stay, and total emergency department (ED) length of stay. ED visit length of stay was defined as the time the patient arrived in the ED until the time they were either discharged or referred by the emergency room physician. Total ED length of stay was defined as the time the patient arrived in the ED until the time they left the ED, either after discharge by the emergency physician or a consulting service, or after being admitted.

\section{Results}

\section{Characteristics of study patients}

Between January and December 2008 a total of 1,148 patients with atrial fibrillation/flutter were seen across the three sites. A total of 451 charts were retrospectively reviewed. Table 1 summarizes the patient characteristics.

\section{Intra-rater reliability}

The kappa statistics for troponin results, presentation of chest pain, altered mental status, heart rate, hypotension, and treatment for acute coronary syndrome by consulting services were $0.9,1.0,0.89,0.89,0.64$ and 1.0 , respectively.

\section{Pattern of troponin testing and results}

A total of 388 (86\%) of patients had at least one set of troponin ordered. In those, $13.7 \%$ had positive troponin results, and $4.9 \%$ were treated as ACS. Forty percent of the patients $(184 / 451)$ were kept for two or more sets of troponins; of those, $26.6 \%(49 / 184)$ had positive troponins, and $9.7 \%(18 / 184)$ were treated for ACS by the consulting service (summarized in Table 2).

The median troponin value was $0.61 \mathrm{ug} / \mathrm{L}(0.77,1.19$, $0.58 \mathrm{ug} / \mathrm{L}$, respectively). Of all patients with positive troponins, $76.7 \%$ had other potential diagnoses for a rise in troponin other than ACS (demand-related rise in troponin $35.7 \%$, congestive heart failure $28.5 \%$, renal failure $16.6 \%$, sepsis $14.3 \%$, pneumonia $2.3 \%$ ).

The percentage of patients with positive troponins who underwent in-hospital workup for acute coronary syndrome, such as stress testing and/or angiograms, was $28.3 \%$ (15/53), with positive results in $46.7 \%(7 / 15)$.

\section{Referral patterns and ED length of stay}

A total of $55 \%$ of patients were referred to consulting services. Those with positive troponins were referred 98\% of the time to consulting services. The median ED visit and ED total length of stay for two sets of troponins versus patients who were not kept for more than 
Table 1 Study and patient population characteristics

\begin{tabular}{|c|c|c|c|c|}
\hline & AED1 & AED2 & CED & Total \\
\hline Total ED visits Jan 012008 - Dec 312008 & 75,500 & 57,300 & 63,000 & 195,800 \\
\hline Total visits with $\mathrm{AF}$ as $1^{\circ}$ or $2^{\circ} \mathrm{Dx}$ & 473 & 271 & 404 & 1,148 \\
\hline \multicolumn{5}{|l|}{ Patient characteristics (\%) } \\
\hline & $N=150$ & $N=151$ & $N=150$ & $N=451$ \\
\hline \multicolumn{5}{|l|}{ Age } \\
\hline$<50$ & $29(19.3)$ & $22(14.6)$ & $18(12.0)$ & $69(15.3)$ \\
\hline $51-60$ & $27(18)$ & $30(19.9)$ & $16(10.7)$ & $73(16.2)$ \\
\hline $61-74$ & $27(18.0)$ & $39(25.8)$ & $45(30.0)$ & $111(24.6)$ \\
\hline$\geq 75$ & $67(44.7)$ & $60(39.7)$ & $71(47.3)$ & $198(43.9)$ \\
\hline \multicolumn{5}{|l|}{ Gender } \\
\hline Male & 89 (59.3) & $98(64.9)$ & $72(48.0)$ & $259(57.4)$ \\
\hline Female & $61(40.7)$ & $53(35.1)$ & $78(52.0)$ & $192(42.6)$ \\
\hline \multicolumn{5}{|l|}{ Type of AF } \\
\hline New onset & $51(34.0)$ & $51(33.8)$ & $36(24.2)$ & $138(30.7)$ \\
\hline Paroxysmal & $92(61.3)$ & $93(61.6)$ & $94(63.1)$ & $279(62.0)$ \\
\hline Persistent & $7(4.7)$ & $6(4.0)$ & $9(6.0)$ & $22(4.9)$ \\
\hline Unknown & $0(0)$ & $1(0.7)$ & $10(6.7)$ & $11(2.4)$ \\
\hline \multicolumn{5}{|l|}{ Duration of symptoms } \\
\hline$<48 \mathrm{~h}$ & $91(60.7)$ & $93(61.6)$ & $100(67.1)$ & $284(63.1)$ \\
\hline $48 \mathrm{~h}$ & $56(37.3)$ & $51(33.8)$ & $33(22.2)$ & $140(31.1)$ \\
\hline Unknown & $3(2.0)$ & $7(4.6)$ & $16(10.7)$ & $26(5.8)$ \\
\hline \multicolumn{5}{|l|}{ Past medical history } \\
\hline History of CHF or EF < 35\% & $40(26.7)$ & $30(19.9)$ & $10(6.7)$ & $80(17.9)$ \\
\hline HTN & $91(60.7)$ & $80(53.0)$ & $98(65.8)$ & $269(59.8)$ \\
\hline CAD & $49(32.7)$ & $31(20.5)$ & $43(28.9)$ & $123(27.3)$ \\
\hline $\mathrm{DM}$ & $20(13.3)$ & $19(12.7)$ & $30(20.1)$ & $69(15.4)$ \\
\hline Previous stroke/TIA & $21(14.0)$ & $23(15.2)$ & $28(18.8)$ & $72(16.0)$ \\
\hline Prosthetic heart valve & $11(7.3)$ & $7(4.6)$ & $5(3.4)$ & $23(5.1)$ \\
\hline \multicolumn{5}{|l|}{ CHADS2 Score* } \\
\hline 0 & $41(23.7)$ & $45(29.8)$ & $29(19.3)$ & $115(25.5)$ \\
\hline 1 & $25(16.7)$ & $43(28.5)$ & $40(26.7)$ & $108(23.9)$ \\
\hline 2 & $34(22.7)$ & $34(22.5)$ & $39(26.0)$ & $107(23.7)$ \\
\hline$\geq 3$ & $50(33.3)$ & 29 (19.2) & $42(28.0)$ & $121(26.8)$ \\
\hline
\end{tabular}

*1 point each for congestive heart failure, hypertension, age $>75$ years, diabetes, and 2 points for transient ischemic attack or stroke

Table 2 Troponin testing and results

\begin{tabular}{ll}
\hline & Total \\
\hline At least one troponin ordered & $N=451$ \\
\hline Positive troponins & $388(86 \%)$ \\
\hline & $53(13.7 \%)$ \\
\hline Treated as ACS & $19(4.9 \%)$ \\
\hline$\geq 2$ Troponins ordered & $184(40.8 \%)$ \\
\hline Positive troponin & $49(26.6 \%)$ \\
\hline Treated as ACS & $18(9.7 \%)$ \\
\hline
\end{tabular}

one set of troponins were 374 and $289.5 \mathrm{~min}$, and 808 and $375.5 \mathrm{~min}$, respectively.

\section{Conclusions}

Our study identified that a high percentage of patients with atrial fibrillation/flutter is kept in the emergency department for troponin testing. Eighty-six percent of patients had at least one set of troponins ordered, and $40 \%$ were kept for two or more sets. Our result is similar to another study, where $42.7 \%$ of patients were kept for a "rule-out myocardial infarction" protocol consisting of three sets of CK-MB cardiac markers [9]. 
In a recent Canadian study, 16.7\% (range 10-27\%) of patients with recent onset atrial fibrillation were admitted from the emergency department [10]. Admission rates were higher (55\%) in our patient population; however, our study included all patients with atrial fibrillation. In our study, $14.7 \%$ of all those with at least one set of troponins and $26.6 \%$ of those with two or more sets had positive troponins. Of importance, $98 \%$ of patients with positive troponins were referred on to consulting services; however, only a third were treated as ACS by consulting services ( $4.9 \%$ of the entire patient cohort). It is possible that patients with positive troponins who were not treated as ACS could have required admission for other reasons; however, our study did not account for that.

The findings of our study have important implications for clinical practice and resource utilization. Cardiac troponins, while relatively cardiac-specific, are not diseasespecific and can be positive in the absence of infarction [11]. For example, in a study of 1,000 consecutive patients who presented to an urban emergency department with potential symptoms or signs of coronary ischemia, $45 \%$ of patients with significantly elevated troponin I levels had a final diagnosis other than ACS [12]. In our study, 79\% of patients with a positive troponin had a potential alternative diagnosis for a rise in troponin other than ACS, corroborating findings from other studies [13]. Demand-related rise in troponin could account for $35.7 \%$ of cases, as has been seen in numerous other studies [14-17]. In a study of patients presenting with elevated TnI levels with subsequent normal coronary angiogram, tachyarrythmias were the cause of TnI release in $28 \%$ of cases [18]. Failure to consider these other potential causes of elevated troponin can lead to unnecessary and invasive cardiac investigations and resource utilization, which will become of more importance with the introduction of newer, more sensitive troponin assays [19]. While it was not within the scope of this study, our work raises interesting questions about the possibility of identifying patients with specific symptoms or characteristics who warrant further diagnostic workup versus patients who are safe to discharge home. These types of questions are best answered in a prospective study, for which our work sheds light on important characteristic in the study population.

Our study has a few limitations. There was no gold standard to establish the diagnosis of acute coronary syndrome, and since the diagnosis of acute coronary syndrome given by cardiologists (and internists) can be open to interpretation, we cannot say with certainty that those who were treated for ACS truly required the treatment. Further, due to the design of our study, we did not look at adverse events in patients who were and were not treated for ACS. There are also inherent limitations of a retrospective chart review, such as the lack of clinical homogeneity among the different sites, missing clinical data, and variability in data abstraction. Specifically, the intra-rater reliability was lower for hypotension compared to other variables, as reflected in the lower kappa statistic. This is likely related to the fact that while even the data abstraction protocols stated that the 'presenting' blood pressure be measured, we did not explicitly define that this blood pressure should be the triage blood pressure, and not any other record. This could have led to confusion regarding which blood pressure was actually recorded. Unfortunately, this was not picked up during our pilot study and random checks. Despite this, we feel our results are sufficiently robust given that a kappa of 0.64 still reflects "substantial agreement," and all other kappa values were high.

In summary, a high percentage of patients with atrial fibrillation/flutter are kept in Canadian emergency departments for troponin testing, and $4.9 \%$ of the total cohort was treated for acute coronary syndrome. Further prospective studies are required to study the clinical implications and prognosis of patients with positive troponins in patients with atrial fibrillation/flutter and to identify those patients who should be tested for troponin and be treated for acute coronary syndrome.

\section{Acknowledgements}

We thank Dr. James Maskalyk for his kind editorial support for the manuscript. Funding Sources/Grants

2009/2010 Summer Medical Student Scholarships of the Heart and Stroke Foundation of Ontario (Emily Austin).

\section{Author details}

'Department of Medicine, Division of Emergency Medicine, University of Toronto, Toronto, Canada 2Queen's University, Kingston, Canada ${ }^{3}$ Department of Family and Community Medicine, University of Toronto, Toronto, Canada ${ }^{4}$ Division of Emergency Medicine, Shahid Beheshti University, Tehran, Iran ${ }^{5}$ University of Toronto, Toronto, Canada ${ }^{6}$ Shahid Beheshti University, Tehran, Iran ${ }^{7}$ York Central Hospital, Toronto, Canada

\section{Authors' contributions}

NM conceived the study, designed the trial, obtained funding, supervised the conduct of the trial and data collection, and drafted the manuscript. EA was a data abstractor, helped with literature review, and edited the first draft of the manuscript. RM provided statistical advice and analyzed the data. $\mathrm{HH}$ helped with data abstraction. AA helped with data abstraction. $\mathrm{BH}$ and $\mathrm{AM}$ were data abstractors.

\section{Competing interests}

The authors declare that they have no competing interests.

Received: 19 January 2011 Accepted: 8 June 2011

Published: 8 June 2011

\section{References}

1. ATRIA Investigators: Prevalence of diagnosed atrial fibrillation in adults. National implications for rhythm management and stroke prevention: the Anticoagulation and Risk Factor in Atrial Fibrillation (ATRIA) Study. JAMA 2001, 285(18):2370-2375.

2. Kannel WB, Wolf PA, Benjamin EJ, Levy D: Prevalence, incidence, prognosis, and predisposing conditions for atrial fibrillation: populationbased estimates. Am J Cardiol 1998, 82(8A):2N-9N.

3. Miyasaka Y, Barnes ME, Gersh BJ, Cha SS, Bailey KR, Abhayaratna WP, Seward JB, Tsang TSM: Secular trends in incidence of atrial fibrillation in 
Olmsted County, Minnesota, 1980 to 2000, and implications on the projections for future prevalence. Circulation 2006, 114:119-25.

4. The Atrial Fibrillation Follow-up Investigation of Rhythm Management (AFFIRM) Investigators: A comparison of rate control and rhythm control in patients with atrial fibrillation. NEJM 2002, 347:1825-1833.

5. Wattigney WA, Mensah GA, Croft JB: Increasing trends in hospitalization for atrial fibrillation in the United States, 1985 through 1999. Circulation 2003, 109:711-716

6. Upshur REG, Moineddin R, Crighton EJ, Mamdani M: Is there a clinically significant seasonal component to hospital admissions for atrial fibrillation? BMC Health Serv Res 2004, 4:5.

7. American College of Cardiology/American Health Association Task Force on practice guideline and the European Society of Cardiology Committee for Practice Guidelines: ACC/AHA/ESC 2006 Guidelines for the management of patients with atrial fibrillation â€" executive summary. Eur Health J 2006, 27:1979-2030.

8. The Task Force for the Management of Atrial Fibrillation of the European Society of Cardiology: Guidelines for the management of atrial fibrillation: Eur Heart J 2010, 31:2369-2429.

9. Zimetbaum PJ, Josephson ME, McDonald MJ, McClennen S, Korley V, Ho KKL, Papageorgiou P, Cohen DJl: Incidence and predictors of myocardial infarction among patients with atrial fibrillation. J Am Coll Cardiol 2000, 36:1223-7.

10. Langhan $T$, Lang ES, Clement CM, Brison RJ, Rowe BH, Borgundvaag $B$, Magee K, Stenstrom R, Perry JJ, Birnie D, Wells GA, Xue X, Innes G, Stiell IG: Predictors of hospitalization in patients presenting to the emergency department with recent onset atrial fibrillation/flutter [abstract]. CJEM 2010, 12:235.

11. Gupta S, de Lemos JA: Use and misuse of cardiac troponins in clinical practice. Prog Cardiovasc Dis 2007, 50(2):151-165.

12. Ng Sl, Krishnaswamy P, Morrisey R, Clopton P, Fitzgerald R, Maisel AS: Mitigation of the clinical significance of spurious elevations of cardiac troponin I in settings of coronary ischemia using serial testing of multiple cardiac markers. Am J Cardiol 2001, 87:994-9.

13. Gupta S, de Lemos JA: Use and misuse of cardiac troponins in clinical practice. Prog Cardiovasc Dis 2007, 50(2):151-165.

14. Zellweger MJ, Schaer BA, Cron TA, Pfisterer ME, Osswald S: Elevated troponin levels in the absence of coronary artery disease after supraventricular tachycardia. Swiss Medical Weekly 2003, 133:439-41.

15. Kanjwal K, Imran N, Grubb B, Kanjwal Y: Troponin elevation in patients with various tachycardias and normal epicardial coronaries. Indian Pacing Electrophysiol J 2008, 8(3):172-4.

16. Nunes JP, Silva JC, Maciel MJ: Troponin I in atrial fibrillation with no coronary atherosclerosis. Acta Cardiol 2004, 59(3):345-6.

17. Miranda RC, Machado MN, Takakura IT, de Mata PF, da Fonseca CGB, Mouco OMCC, Hernandes ME, Lemos MABT, Maia LN: Elevated troponin levels after prolonged supraventricular tachycardia in patient with normal coronary angiography. Cardiology 2006, 106(1):10-13.

18. Bakshi TK, Choo MKF, Edwards CC, Scott AG, Hart HH, Armstrong GP: Causes of elevated troponin I with a normal coronary angiogram. Int Med J 2002, 32:520-5.

19. Keller T, Zeller T, Peetz D, Tzikas S, Roth A, Czyz E, Bickel C, Blds S, Warnholtz A, Frohlich M, Sinning CR, Eleftheriadis MS, Wild PS, Schnabel RB, Lubos E, Jahmann N, Genth-Zotz S, Post F, Nicaud V, Tiret L, Lackner KJ, Munzel TF, Blankenberg S: Sensitive troponin I assay in early diagnosis of acute myocardial infarction. NEJM 2009, 361(9):868-877.

doi:10.1186/1865-1380-4-25

Cite this article as: Meshkat et al.: Troponin utilization in patients presenting with atrial fibrillation/flutter to the emergency department: retrospective chart review. International Journal of Emergency Medicine 2011 4:25.

\section{Submit your manuscript to a SpringerOpen ${ }^{\circ}$ journal and benefit from:}

- Convenient online submission

- Rigorous peer review

- Immediate publication on acceptance

- Open access: articles freely available online

- High visibility within the field

- Retaining the copyright to your article

Submit your next manuscript at $>$ springeropen.com 